The next step for our study will be direct determination, such as faecal $H$. pylori antigens or $H$. pylori determination in BAL (i.e. DNA PCR or culture), in a wide population of IPF patients. The effects of IPF pharmacological treatments on $H$. pylori infection remain to be established.

@ERSpublications

Helicobacter pylori infection in IPF patients is associated with higher rates of mortality and PFTs decline http://ow.ly/qKb3K

David Bennett ${ }^{1}$, Elena Bargagli ${ }^{1}$, Rosa Metella Refini ${ }^{1}$, Maria Stella Campagna ${ }^{2}$, Luigi Gennari ${ }^{2}$, Ranuccio Nuti ${ }^{2}$, Natale Figura ${ }^{2}$ and Paola Rottoli ${ }^{1}$

${ }^{1}$ Respiratory Diseases Unit, Dept of Medical and Surgical Sciences \& Neurosciences, University of Siena, Siena, and

${ }^{2}$ Internal Medicine Unit, Dept of Medical and Surgical Sciences \& Neurosciences, University of Siena, Siena, Italy.

Correspondence: D. Bennett, Respiratory Diseases Unit, Medical and Surgical Sciences \& Neurosciences, University of Siena, "Le Scotte" Hospital, Viale Bracci, 16, 53100, Siena, Italy. E-mail: david.btt@gmail.com

Received: June 192013 | Accepted after revision: Sept 192013 | First published online: Oct 102013

Conflict of interest: None declared.

\title{
References
}

Ibrahim WH. Helicobacter pylori eradication in the management of idiopathic pulmonary fibrosis. Eur Respir $J$ 2007; 30: 395-396.

2 Raghu G, Freudenberger TD, Yang S, et al. High prevalence of abnormal acid gastro-oesophageal reflux in idiopathic pulmonary fibrosis. Eur Respir J 2006; 27: 136-142.

3 Savarino E, Carbone R, Marabotto E, et al. Gastro-oesophageal reflux and gastric aspiration in idiopathic pulmonary fibrosis patients. Eur Respir J 2013; 42: 1322-1331.

4 Kanbay M, Kanbay A, Boyacioglu S. Helicobacter pylori infection as a possible risk factor for respiratory system disease: a review of the literature. Respir Med 2007; 101: 203-209.

5 Figura N, Gennari L, Merlotti D, et al. Prevalence of Helicobacter pylori infection in male patients with osteoporosis and controls. Dig Dis Sci 2005; 50: 847-852.

Bhandari A, Crowe SE. Helicobacter pylori in gastric malignancies. Curr Gastroenterol Rep 2012; 14: 489-496.

Suzuki H, Franceschi F, Nishizawa T, et al. Extragastric manifestations of Helicobacter pylori infection. Helicobacter 2011; 16: Suppl. 1, 65-69.

8 Gencer M, Ceylan E, Yildiz Zeyrek F, et al. Helicobacter pylori seroprevalence in patients with chronic obstructive pulmonary disease and its relation to pulmonary function tests. Respiration 2007; 74: 170-175.

9 Zhuo WL, Zhu B, Xiang ZL, et al. Assessment of the relationship between Helicobacter pylori and lung cancer: a meta-analysis. Arch Med Res 2009; 40: 406-410.

10 Fullerton D, Britton JR, Lewis SA, et al. Helicobacter pylori and lung function, asthma, atopy and allergic disease - a population-based cross-sectional study in adults. Int J Epidemiol 2009; 38: 419-426.

11 Raghu G, Collard HR, Egan JJ, et al. An official ATS/ERS/JRS/ALAT Statement: idiopathic pulmonary fibrosis: evidence-based guidelines for diagnosis and management. Am J Respir Crit Care Med 2011; 183: 788-824.

12 Gonnelli S, Caffarelli C, Tanzilli L, et al. The associations of body composition and fat distribution with bone mineral density in elderly Italian men and women. J Clin Densitom 2013; 16: 168-177.

13 Hogaboam, C. Pathogenesis of IPF. The 6th WASOG Conference, June 6th-7th 2013, Paris, France. www. wasog2013.com/Program Date last accessed: November 5, 2013.

\section{Combined pulmonary fibrosis and emphysema syndrome associated with ABCA3 mutations}

To the Editor:

Herein, we present the first report of combined pulmonary fibrosis and emphysema (CPFE) in an adult patient who was compound heterozygous for mutations of the ATP-binding cassette subfamily A member 3 gene (ABCA3, MIM 601615).

A 41-year-old nonsmoking male presented with dyspnoea on mild exertion. The patient's medical history indicated neonatal respiratory distress, gastro-oesophageal reflux and pneumonia 8 years previously that resolved with antibiotics. His physical examination revealed a mild pectus excavatum, finger clubbing and bilateral basal crackles. High-resolution computed tomography (HRCT) of the chest showed voluminous 
emphysema in the upper zones of the lungs associated with honeycomb fibrosis and ground-glass opacity in lower lobes, predominating in left lung (fig. 1). The bronchoalveolar lavage differential cell count was $67 \%$ macrophages, $22 \%$ neutrophils and $8 \%$ lymphocytes. Pulmonary function tests showed: total lung capacity of $75 \%$, vital capacity (VC) of $50 \%$, residual volume of $134 \%$; forced expiratory volume in $1 \mathrm{~s}(\mathrm{FEV} 1)$ of $49 \%$, diffusing capacity of the lung for carbon monoxide of $38 \%$ predicted, $\mathrm{FEV} 1 / \mathrm{VC}$ of $74 \%$, and arterial oxygen tension at room air was $96 \mathrm{mmHg}$. During a 6-min walk test the peripheral oxygen saturation decreased from $96 \%$ at rest to $90 \%$ after $630 \mathrm{~m}$ (80\% of predicted value). A lung biopsy was not performed. Doppler echocardiography showed normal heart cavities, with estimated systolic pulmonary arterial pressure of $37 \mathrm{mmHg}$. Serum $\alpha_{1}$-antitrypsin levels, autoimmune markers (including anti-nuclear antibody and rheumatoid factor), and immunoglobulin pattern were normal.

This clinical presentation of CPFE in a young patient prompted us to screen mutations in genes causing surfactant dysfunction. After informed consent was obtained, sequencing analysis of the surfactant protein C gene (SFTPC, MIM 178620) revealed no mutation. Sequence analysis of the ABCA3 gene identified two mutations: 1) c.3081_3092delinsCG resulting in a serine to valine change at codon 1028 with the creation of a stop codon 103 amino acids downstream (p.Ser1028Valfs ${ }^{\star} 103$ ); and 2) the common mutation c.875A $>$ T changing a glutamic acid to a valine at codon 292 (p.Glu292Val). None of these mutations were found in either the public polymorphism database or our controls. 2 years after presentation, chest HRCT as well as lung function worsened and azithromycin ( $250 \mathrm{mg}$ every other day) was initiated.

Pulmonary surfactant, a complex mixture of lipids and specific proteins located at the air-liquid interface, lowers alveolar surface tension thereby preventing alveolar collapse at the end of expiration. It is synthesised by alveolar type II cells, stored in lamellar bodies, and secreted by exocytosis. ABCA3 is expressed in the lamellar bodies of alveolar type II cells and is crucial to pulmonary surfactant storage and homeostasis. Several studies indicated a role of genes involved in surfactant metabolism in the development of diffuse lung diseases [1].

CPFE is a syndrome characterised by the coexistence of emphysema and pulmonary fibrosis in the same patient [2]. It typically occurs in male smokers and is associated with dyspnoea, upper lobe emphysema, lower lobe fibrosis and abnormalities of gas exchange. In the absence of the SFTPC mutation, previously associated with CPFE [3], we decided to analyse other genes involved in surfactant metabolism, such as $A B C A 3$. Recessive loss-of-function mutations in $A B C A 3$ present as lethal surfactant deficiency in the newborn, whereas other recessive mutations in $A B C A 3$ can result in interstitial lung disease in older children [4]. Previous studies showed that homozygous or compound heterozygous $A B C A 3$ mutations led
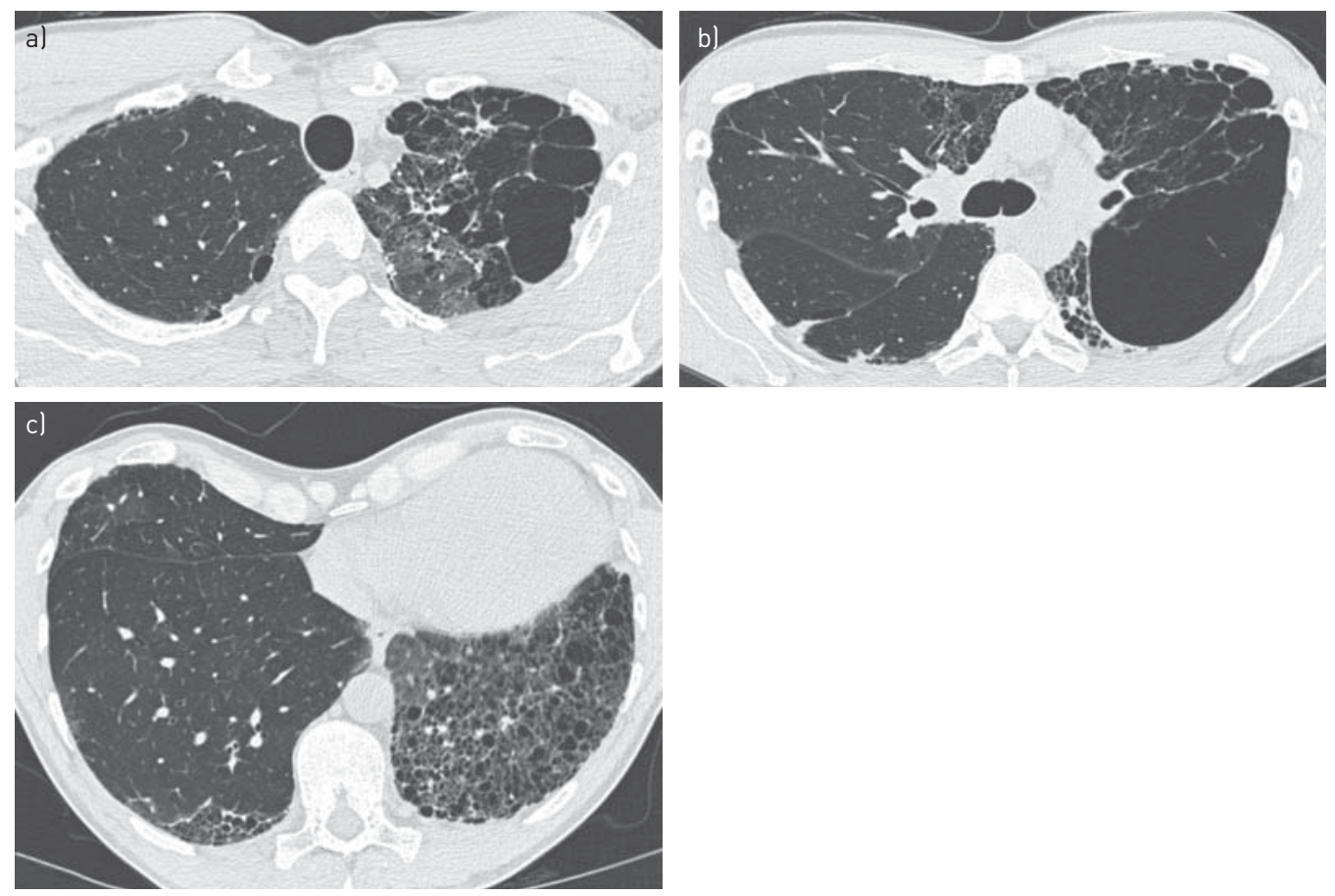

FIGURE 1 High-resolution computed tomography of the chest showing a, b) left upper lobe predominant emphysema associated with a, c) ground-glass opacities and c) asymmetric honeycomb pattern in the lower zones. 
to abnormal processing and/or trafficking of the ABCA3 protein [5], alterations in $\mathrm{ABCA} 3$ protein functions such as ATPase activity [6], or impaired lipid transport [7]. As previously described, our patient had a less severe phenotype than is usually associated with $A B C A 3$ mutations [4]. These variations in the clinical and radiological features may be related to the nature of the mutation. Our patient was found to be compound heterozygous for $A B C A 3$ mutations. The first is the common mutation p.Glu292Val, which is found in heterozygous form with a frequency of $<1 \%$ [8] and has been previously reported to be associated with mild lung disease. The second has not yet been described but is expected to be a disease-causing mutation as it introduces a premature termination codon, likely to be associated with markedly reduced mRNA levels due to nonsense-mediated degradation. Such a "null" allele precludes any functional ABCA3 from being made resulting in abnormal lamellar bodies, but should be less deleterious in combination with the mild mutation p.Glu292Val. Interactions with variants in other genes and/or with external factors such as viral or bacterial infections, as observed in our case, may also influence the observed phenotype [9].

The phenotype of our patient is very similar to that observed in the case reported by COTTIN et al. [3] carrying SFTPC mutations. Our patient is a nonsmoker and the emphysematous lesions were voluminous and localised, mostly in the upper lobes, whereas asymmetric fibrosis lesions were predominant in the lower lobes.

There is no specific treatment for CPFE syndrome. Supported immunosuppressive therapy was not indicated in this case without evidence of active inflammation. Improvement of severe interstitial lung disease in a young patient with ABCA3 deficiency has been reported after treatment with azithromycin, an azalide macrolide antibiotic characterised by a nitrogen in the macrolide ring [10]. Although, there is no evidence of efficacy of azithromycin in CPFE, the worsening of our patient's respiratory status together with the safety of this drug incited us to initiate this treatment in our patient.

To our knowledge, this is the first report of a phenotype of CPFE syndrome in an adult patient carrying mutations of the $A B C A 3$ gene. Although further studies are needed to confirm the role of surfactant metabolism in CPFE, this result suggests that this syndrome may have an underlying genetic predisposition.

0 @ERSpublications

The first report of combined pulmonary fibrosis and emphysema in an adult carrying compound heterozygote ABCA3 mutations http://ow.ly/rm0Dd

Ralph Epaud ${ }^{1,2,3}$, Céline Delestrain ${ }^{1}$, Malek Louha ${ }^{4}$, Stéphanie Simon ${ }^{1,2}$, Pascale Fanen ${ }^{1,2}$ and Abdellatif Tazi ${ }^{5,6}$

${ }^{1}$ Inserm, U955, Equipe 11, Créteil, ${ }^{2}$ Université Paris-Est, UMR_S955, UPEC, Créteil, ${ }^{3}$ Centre Hospitalier Intercommunal de Créteil, Service de Pédiatrie, Créteil, ${ }^{4}$ AP-HP, Hôpital Armand Trousseau, Service de Biochimie-Génétique Moléculaire, Paris, ${ }^{5}$ Université Paris Diderot, Paris, and ${ }^{6}$ INSERM UMR 717, AP-HP, Service de Pneumologie, Hôpital Saint Louis, Paris, France.

Correspondence: R. Epaud, Service de Pédiatrie, Centre Hospitalier Intercommunal, 40 avenue de Verdun, 94000 Créteil, France. E-mail: ralph.epaud@chicreteil.fr

Received: Aug 192013 | Accepted after revision: Sept 212013 | First published online: Oct 172013

Support statement: This work was supported by INSERM (P. Fanen, R. Epaud and C. Delestrain), Assistance PubliqueHôpitaux de Paris (A. Tazi) and the Société de Pneumologie de Langue Française (C. Delestrain). ABCA3 gene analysis technic was designed and initiated for Surfactant Disorders and Chronic Lung Disease (APSE; ClinicalTrials.gov identifier NCT00783978) (R. Epaud and M. Louha).

Conflict of interest: None declared.

\section{References}

Deutsch GH, Young LR, Deterding RR, et al. Diffuse lung disease in young children: application of a novel classification scheme. Am J Respir Crit Care Med 2007; 176: 1120-1128.

2 Cottin V, Nunes H, Brillet PY, et al. Combined pulmonary fibrosis and emphysema: a distinct under recognised entity. Eur Respir J 2005; 26: 586-593.

3 Cottin V, Reix P, Khouatra C, et al. Combined pulmonary fibrosis and emphysema syndrome associated with familial SFTPC mutation. Thorax 2011; 66: 918-919.

4 Flamein F, Riffault L, Muselet-Charlier C, et al. Molecular and cellular characteristics of ABCA3 mutations associated with diffuse parenchymal lung diseases in children. Hum Mol Genet 2011; 21: 765-775.

5 Cheong N, Madesh M, Gonzales LW, et al. Functional and trafficking defects in ATP binding cassette A3 mutants associated with respiratory distress syndrome. J Biol Chem 2006; 281: 9791-9800.

6 Matsumura Y, Ban N, Ueda K, et al. Characterization and classification of ATP-binding cassette transporter ABCA3 mutants in fatal surfactant deficiency. J Biol Chem 2006; 281: 34503-34514.

7 Matsumura Y, Ban N, Inagaki N. Aberrant catalytic cycle and impaired lipid transport into intracellular vesicles in ABCA3 mutants associated with nonfatal pediatric interstitial lung disease. Am J Physiol Lung Cell Mol Physiol 2008; 295: L698-L707. 
8 Wambach JA, Wegner DJ, Depass K, et al. Single ABCA3 mutations increase risk for neonatal respiratory distress syndrome. Pediatrics, 130: e1575-e1582.

9 Bullard JE, Nogee LM. Heterozygosity for ABCA3 mutations modifies the severity of lung disease associated with a surfactant protein C gene (SFTPC) mutation. Pediatr Res 2007; 62: 176-179.

10 Thouvenin G, Nathan N, Epaud R, et al. Diffuse parenchymal lung disease caused by surfactant deficiency: dramatic improvement by azithromycin. BMJ Case Rep 2013 [in press DOI: 10.1136/bcr-2013-009988].

\title{
Pulmonary arterial hypertension in familial hemiplegic migraine with ATP1A2 channelopathy
}

\begin{abstract}
To the Editor:
Pulmonary arterial hypertension (PAH) has been the focus of major research in recent years [1]. Involvement of mutations in genes encoding for members of the transforming growth factor- $\beta$ signalling pathway (BMPR2, ACVRL1, ENG and SMAD8) has been demonstrated in the development of heritable $\mathrm{PAH}$, allowing novel experimental and clinical approaches [2-4]. However, $\sim 30 \%$ of familial forms of PAH remain without any identification of genetic mutations. Recently, mutations of the KCNK3 gene (encoding $\mathrm{K}^{+}$channel subfamily $\mathrm{K}$ member 3 ) have been reported in patients with familial and sporadic PAH [5]. KCNK3 belongs to a family of mammalian $\mathrm{K}^{+}$channels, and are involved in the regulation of resting membrane potential, pulmonary vascular tone and in vascular remodelling. This result paves the way to the involvement of novel signalling pathways in the development of heritable PAH. Herein, we describe a novel association of PAH and a channelopathy due to mutation in ATP1A2 (encoding the $\alpha 2$-subunit of the $\mathrm{Na}^{+} / \mathrm{K}^{+}$-ATPase), a mutation known to cause familial hemiplegic migraine (FHM), a rare autosomal dominant disease [6].
\end{abstract}

A 24-year-old male was referred with a 1-year history of progressive exertional dyspnoea. Since the age of 8 years, he has reported recurrent episodes of hemiplegic migraine associated with muscle weakness and pain. The proband's mother (II4) (fig. 1) and two of his brothers (III6 and III7) had recurrent hemiplegic migraine with aura. There was no familial history of PAH. On admission, the patient was in New York Heart Association (NYHA) functional class III. His 6-min walk distance (6MWD) was 409 m. Pulmonary function tests were normal except for decreased diffusing capacity of the lungs for carbon monoxide. Doppler transthoracic echocardiography revealed signs of severe pulmonary hypertension with an estimated systolic pulmonary artery pressure of $75 \mathrm{mmHg}$, right ventricular dilatation and hypertrophy, and mild pericardial effusion. Right heart catheterisation confirmed pre-capillary pulmonary hypertension, with a mean pulmonary artery pressure $(\mathrm{mPAP})$ of $51 \mathrm{mmHg}$, a pulmonary capillary wedge pressure of $12 \mathrm{mmHg}$, a right atrial pressure of $7 \mathrm{mmHg}$, a cardiac index of $1.90 \mathrm{~L} \cdot \mathrm{min}^{-1} \cdot \mathrm{m}^{-2}$ and pulmonary vascular resistance (PVR) of 12.3 Wood units. No acute vasodilator response to nitric oxide was observed. Screening for other causes of pulmonary hypertension was negative. The patient was treated with a combination of intravenous epoprostenol, an endothelin receptor antagonist (ERA) and a phosphodiesterase type 5 inhibitor (PDE5i). The patient stopped taking the PDE5i after a few days because of side-effects, including increased symptoms of migraine. 4 months later, re-evaluation showed moderate clinical (NYHA functional class II and 6MWD $518 \mathrm{~m}$ ) and haemodynamic improvement (mPAP $43 \mathrm{mmHg}$, cardiac index $2.29 \mathrm{~L} \cdot \mathrm{min}^{-1} \cdot \mathrm{m}^{-2}$ and PVR 8.4 Wood units). The patient is still alive 1 year after diagnosis on intravenous epoprostenol and an ERA.

According to our local procedures, the patient underwent genetic counselling and gave written informed consent for genetic screening. No point mutations or large rearrangements of the BMPR2 and ACVRL1 genes were identified. To date, three genes (CACNA1A, ATP1A2 and SCNA1) encoding ion transporters are known to be associated with FHM. Genetic analysis revealed a nucleotide substitution in the coding sequence of the ATP1A2 gene (c.2819C>T; p.S940L) located on chromosome 1 (1q23). This mutation, which was not found in 200 control chromosomes, and was absent from the dbSNP, 1000 Genomes and Exome Sequencing Project data, affects a highly conserved amino acid, but has never been reported before. The patient's brothers, III4 and III6, were screened for the familial ATP1A2 mutation. Patient III4 did not carry the familial mutation and, as suggested by the clinical symptoms, the mutation was identified in patient III6 (fig. 1). Mutations of the ATP1A2 gene are known to cause FHM, a rare autosomal dominant disease characterised by migraine with motor weakness and aura [6]. Other neurological symptoms include 